\title{
STABILITY ANALYSIS OF RUNGE-KUTTA METHODS APPLIED TO A BASIC VOLTERRA INTEGRAL EQUATION
}

\author{
CHRISTOPHER T. H. BAKER AND JOAN C. WILKINSON
}

(Received 10 July 1980)

(Revised 13 November 1980)

\begin{abstract}
Our purpose in this paper is to display the stability analysis of Runge-Kutta methods applied to a Volterra integral equation of a simple form. As prerequisite we define, and then develop the structure of, the class of Runge-Kutta methods considered. The test equation is taken as the "basic" equation $f(x)-\lambda \int_{0}^{x} f(y) d y=g(x)$; the simple form of this equation permits ready insight into features which are more obscure when considering (as elsewhere [1], [2], [6]) equations of a more complicated form. Due to the structure of the methods and the nature of the test equation, the stability analysis reduces to the study of recurrence relations of the form $\Phi_{k+1}=\mathbf{M} \Phi_{k}+\gamma_{k}(k=0,1,2, \ldots)$ which are common in stability discussions in numerical analysis.
\end{abstract}

\section{Numerical methods and stability}

We consider methods applicable to the numerical solution of the Volterra integral equation

$$
f(x)-\int_{0}^{x} K(x, y, f(y)) d y=g(x), \quad x \geq 0,
$$

wherein the given functions $K(x, y, v)$ and $g(x)$ are continuous on suitable regions; we assume Lipschitz continuity (in $v$ ) for the "kernel function" $K$. The methods we consider are Runge-Kutta methods; they bear an affinity to quadrature methods associated with a family of quadrature rules

$$
\int_{0}^{i h} \phi(y) d y \simeq h \sum_{k=0}^{i} \omega_{i k} \phi(k h) \quad \text { for } i=1,2, \ldots ; h \geqslant 0 .
$$

We define $\omega_{i k}=0$ if $k>i$.

OCopyright Australian Mathematical Society 1981 
Definition 1.1. A quadrature method for (1.1) defined by the rules (1.2) consists of determining values $\tilde{f}(i h), i=1,2,3, \ldots$, such that $\tilde{f}(0)=f(0)=g(0)$ and

$$
\tilde{f}(i h)-h \sum_{k=0}^{i} \omega_{i k} K(i h, k h, \tilde{f}(k h))=g(i h), \quad i=1,2,3, \ldots
$$

There is a conceptual difference (which it would be pedantic to insist upon) between a method and its formulae, and we shall refer, for example, to the formulae (1.3) as a quadrature method defined by (1.2). By the same token we shall later describe a method, applied to a test problem, as stable when the resulting recurrence relations are stable.

\subsection{Mixed and extended Runge-Kutta methods}

In quadrature methods, we determine in "step-by-step" fashion the values $\tilde{f}(h), \tilde{f}(2 h), \tilde{f}(3 h), \ldots$ More general methods involve the selection of $\boldsymbol{\theta}_{0}$, $\theta_{1}, \ldots, \theta_{p-1}$ and $\theta_{p}=1$ and the calculation of blocks of related values $\tilde{f}_{j}$ approximating $f(x)$ at points

$$
\tau_{j}=i h+\theta_{r} h, \quad r=0,1, \ldots, p,
$$

successively for $i=0,1,2, \ldots$, with $j=i(p+1)+r+1$ and $\tilde{f}_{0}=g(0)$. The blocks of values define successive vectors

$$
\boldsymbol{\phi}_{i+1}=\left[\tilde{f}_{i(p+1)+1}, \tilde{f}_{i(p+1)+2}, \ldots, \tilde{f}_{(i+1)(p+1)}\right]^{T}, \quad i>0 .
$$

Amongst such methods are the Runge-Kutta methods of interest to us here ${ }^{2}$. To avoid confusion when the points $\left\{\tau_{j}\right\}$ are not distinct, we write $\tilde{f}_{j}$ rather than $\tilde{f}\left(i h+\theta_{r} h\right)$. Observe that $r \equiv(j-1) \bmod (p+1)$ in equation (1.4).

The principal purpose of the Runge-Kutta methods may be considered to be the determination of approximations for $f(h), f(2 h), f(3 h), \ldots$ The required approximations are found, since $\theta_{p}=1$, as

$$
\tilde{f}(i h)=\tilde{f}_{i(p+1)}, \quad i=0,1,2, \ldots
$$

The intermediate values $\tilde{f}_{i(p+1)+s}, s \neq 0 \bmod (p+1)$, may also be of interest [9].

The Runge-Kutta methods under discussion correspond to a choice of $\theta_{0}$, $\theta_{1}, \ldots, \theta_{p-1}$ and a set of formulae

$$
\int_{0}^{\tau_{j}} \phi(y) d y \simeq h \sum_{k>0} \Omega_{j k} \phi\left(\tau_{k}\right), \quad j=1,2,3, \ldots,
$$

\footnotetext{
${ }^{1}$ We here assume the formulae will be solved exactly.

2 See also [5]. More general methods have been proposed by Beltyukov [4], [11], van der Houwen [17] and Weiss (see [4]).
} 
which, applied to discretize (1.1) with $x=\tau_{j}$, yield equations

$$
\tilde{f}_{j}-h \sum_{k>0} \Omega_{j k} K\left(\tau_{j}, \tau_{k}, \tilde{f}_{k}\right)=g\left(\tau_{j}\right), \quad j=1,2,3, \ldots
$$

We suppose that

$$
\Omega_{j k}=0 \text { if }[(k-1) /(p+1)]>i=[(j-1) /(p+1)],
$$

where $[z]$ denotes the integer part of $z$. The methods $(1.7 \mathrm{a}, \mathrm{b})$ appear as natural extensions of the quadrature methods. In Runge-Kutta methods the choice of parameters $\left\{\theta_{j}\right\}\left\{\Omega_{j k}\right\}$ is motivated by questions of order of accuracy, and we offer the following definition.

Definition 1.2. A method (1.7a, b) is a Runge-Kutta method if the values (1.5) display superconvergence as $h \rightarrow 0$, with ih fixed, for a suitably restricted set of problems (1.1).

REMARK. If the values $\tau_{j}, j=0,1,2, \ldots$, contain repetitions it is not, in general, possible to define a single-valued function $\tilde{f}$ on the mesh $\left\{\tau_{j}\right\}$ with $\tilde{f}\left(\tau_{j}\right)=\tilde{f}$. Therefore, the reader may wish to assume that $\theta_{0}, \theta_{1}, \ldots, \theta_{p-1}$ are distinct points in $(0,1)$ and we may then write $\tilde{f}\left(\tau_{j}\right)=\tilde{f}_{j}$ and

$$
\tilde{f}\left(\tau_{j}\right)-h \sum_{k>0} \Omega_{j k} K\left(\tau_{j}, \tau_{k}, \tilde{f}\left(\tau_{k}\right)\right)=g\left(\tau_{j}\right) .
$$

We shall not concern ourselves here with questions of order of convergence but shall refer to our methods, described in more detail below, as Runge-Kutta methods. Our intention has been to motivate Runge-Kutta methods for (1.1). Specific methods result from a choice of formulae (1.6), satisfying (1.7b), which we shall here associate with formulae

$$
\int_{0}^{\theta_{t} h} \phi(y) d y \simeq h \sum_{s=0}^{p} A_{t s} \phi\left(\theta_{s} h\right), \quad t=0,1, \ldots, p .
$$

The rules (1.9) correspond to a Runge-Kutta tableau

$$
[\theta \mid \mathrm{\theta}]=\begin{array}{c|llll}
\theta_{0} & A_{00} & A_{01} & \cdots & A_{0 p} \\
\theta_{1} & A_{10} & A_{11} & \cdots & A_{1 p} \\
& & & & \\
\theta_{p-1} & A_{p-1,0} & A_{p-1,1} & & A_{p-1, p} \\
\hline \theta_{p}^{-}=1 & -\frac{A_{p 0}}{A_{p 1}} & \cdots & A_{p p}
\end{array} .
$$

DEFINITION 1.3. A Runge-Kutta array (1.10) occurring (normally with $A_{r p}=0$ for $r=0,1, \ldots, p)$ in the numerical treatment of ordinary differential equations will be called a conventional R-K array. 
We write $\tau_{j}=i h+\theta_{r} h, r \equiv(j-1) \bmod (p+1)$, and (whether or not $\tau_{j}>i h$ )

$$
\int_{0}^{\tau_{j}} \phi(y) d y=\int_{0}^{i h} \phi(y) d y+\int_{i h}^{\tau} \phi(y) d y .
$$

The rules (1.9) applied (a) in repeated form with $t=p$ (b) with $t=r$ yield

$$
\int_{0}^{\tau} \phi(y) d y \simeq h \sum_{k=0}^{i-1} \sum_{s=0}^{p} A_{p s} \phi\left(\tau_{k(p+1)+s+1}\right)+h \sum_{s=0}^{p} A_{r s} \phi\left(\tau_{i(p+1)+s+1}\right),
$$

$\tau_{j}$ being defined above.

Definition 1.4. A Runge-Kutta method $(1.7 \mathrm{a}, \mathrm{b})$ is called an extended Runge-Kutta method if the formulae (1.6) and (1.12) coincide.

We may employ (1.2) to approximate $\int_{0}^{i h} \phi(y) d y$ in (1.11) and obtain, in lieu of (1.12), and setting $\omega_{00}=0$, the formulae

$$
\int_{0}^{\tau_{j}} \phi(y) d y \simeq h \sum_{k=0}^{i} \omega_{i k} \phi\left(\tau_{k(p+1)}\right)+h \sum_{s=0}^{p} A_{r s} \phi\left(\tau_{i(p+1)+s+1}\right) .
$$

Definition 1.5. A Runge-Kutta method $(1.7 \mathrm{a}, \mathrm{b})$ is called a mixed quadrature-Runge-Kutta method if the formulae (1.6) and (1.13) coincide.

1.1.1. Another class of "mixed" methods (considered by Pouzet [21] and of some interest in [9]) arises on identifying (1.6) with formulae

$$
\int_{0}^{\tau} \phi(y) d y \simeq h \sum_{k=0}^{i-1} \sum_{s=0}^{p} B_{p s} \phi\left(\tau_{k(p+1)+s+1}\right)+h \sum_{s=0}^{p} A_{r s} \phi\left(\tau_{i(p+1)+s+1}\right) .
$$

We indicate what we shall call economized versions of the mixed quadratureRunge-Kutta methods, available when $\theta_{0}=0$. In this case, since $\theta_{p}=1, \tau_{i(p+1)}$ $=\tau_{t(p+1)+1}=i h$ and we have two approximations at the point $i h$. Some apparent saving of effort is achieved if we modify the methods of Definition 1.5 by setting $\tilde{f}_{i(p+1)+1}=\tilde{f}_{i(p+1)}=\tilde{f}(i h)$, rather than compute the new value $\tilde{f}_{i(p+1)+1}$. (In general this leads to a new method. Van der Houwen [17] has constructed methods which generalize the economized methods and have interesting properties.) We observe that methods of Beltyukov type [4], [11] reduce to economized methods when applied to the basic test equation which we considered here.

\subsection{Stability}

The study of stability of the solution of the integral equation (1.1) is the study of the sensitivity of $f(x)$ to perturbations in the problem (say in $g(x)$ ) in particular as $x \rightarrow \infty$. For an introduction to this topic we refer to the review of Tsalyuk [23]. The study of stability is not amenable to investigation unless 
restrictions are placed on the class of "admissible" perturbations and on (1.1). When (1.1) is linear, progress in the stability analysis can be made by analyzing the resolvent or differential resolvent [23]. For the basic test equation

$$
f(x)-\lambda \int_{0}^{x} f(y) d y=g(x)
$$

a constant change $\delta$ in $g(x)$ results in a change $\varepsilon(x)=\delta \exp (\lambda x)$ in $f(x)$ (see below) which (a) is bounded if and only if $\operatorname{Re}(\lambda)<0$ and (b) decays to zero as $x \rightarrow \infty$ if and only if $\operatorname{Re}(\lambda)<0$. The consequent classification of (1.15) is embodied in the following definition.

DEFINITION 1.6. Equation (1.15) is stable if $\operatorname{Re}(\lambda) \leqslant 0$ and asymptotically stable if $\operatorname{Re}(\lambda)<0$.

The behaviour of $\varepsilon(x)$ in the preceding discussion follows from the equation $\varepsilon(x)-\lambda \int_{0}^{x} \varepsilon(y) d y=\delta$, which yields

$$
\varepsilon^{\prime}(x)=\lambda \varepsilon(x), \quad \varepsilon(0)=\delta,
$$

on differentiating. On the other hand, equations (1.7a) yield, when applied to (1.15),

$$
\tilde{f}_{j}-\lambda h \sum_{k>0} \Omega_{j k} \tilde{f}_{k}=g_{j}
$$

and we seek the effect of perturbations in the values $g_{j}$ on the values $\tilde{f}_{j}$. Structure in the weights $\Omega_{j k}$ permits us to mirror (via a differencing process) the derivation of (1.16), in order to obtain a finite term recurrence relation from (1.17).

\subsection{Stability of recurrence relations}

A differencing procedure applied to (1.17) will yield, under conditions assumed here, recurrence relations of the form

$$
\boldsymbol{\Phi}_{k+1}=\mathrm{M \Phi}_{k}+\gamma_{k}, \quad k=0,1,2, \ldots,
$$

where

$$
\mathrm{M} \equiv \mathbf{M}(\lambda h)
$$

and $\boldsymbol{\Phi}_{0}=\gamma$ is given. The components of the vectors $\boldsymbol{\Phi}_{k}$ will be successive values $\tilde{f}_{j}$. The study of (1.18) is commonplace in numerical analysis but has interest in its own right (Hahn [14, p. 47], Miller [20]).

A perturbation $\varepsilon$ in $\Phi_{0}$ results in perturbations $\mathbf{M}^{k} \varepsilon$ in $\boldsymbol{\Phi}_{k}$ which certainly decay if the spectral radius $\rho(\mathbf{M})<1$, and are bounded if $\rho(\mathbf{M})=1$ and also $\mathbf{M}$ is $^{3}$ of class $M$. In each case, however, we may find that the norm $\|\mathbf{M} \varepsilon\|$ of the

${ }^{3}$ A matrix is of class $M$ if and only if its eigenvalues of largest modulus are semi-simple (i.e. have equal algebraic and geometric multiplicities). 
change in $\Phi_{1}$ associated with $\varepsilon$ is larger than $\|\varepsilon\|$ unless $\|\mathbf{M}\|<1$ in the particular subordinate norm. (For any $\mathbf{M}, \rho(\mathbf{M})<\|\mathbf{M}\|$.)

DEFINITION 1.7. (a) A recurrence (1.18) is block-stable in the specified norm \| \| if the subordinate norm of the amplification matrix $\mathbf{M}$ satisfies $\|\mathbf{M}\|<1$ and the stability is strict if $\|\mathbf{M}\|<1$. (b) A square matrix is strictly stable if $\rho(\mathbf{M})<1$ and is stable (Varga [24]) if and only if either (i) $\rho(\mathbf{M})<1$ or (ii) $\rho(\mathbf{M})=1$ and $\mathbf{M}$ is of class $M$. A recurrence (1.18) is called (strictly) stable when its amplification matrix is (strictly) stable.

REMARK. A rank-one matrix with non-zero spectral radius is of class $M$.

Recurrences frequently encountered have the form

$$
\sum_{l=0}^{m} \mathbf{X}_{l}(\lambda h) x_{n+1-l}=\gamma_{n+1}^{*}, \quad \operatorname{det}\left[\mathbf{x}_{0}(\lambda h)\right] \neq 0,
$$

which may reduce to a scalar recurrence. We obtain a relation (1.18) on writing $\boldsymbol{\Phi}_{n+1}=\left[\boldsymbol{X}_{n+1}^{T}, \boldsymbol{X}_{n}^{T}, \ldots, \boldsymbol{X}_{n+1-m}^{T}\right]^{T}$ and

$$
\mathbf{M}(\lambda h)=\left[\begin{array}{cccccc}
-\mathbf{X}_{0}^{-1} \mathbf{X}_{1} & & -\mathbf{X}_{0}^{-1} \mathbf{X}_{2} & \cdots & \cdots & -\mathbf{X}_{0}^{-1} \mathbf{X}_{m} \\
\mathbf{I} & & \mathbf{0} & \mathbf{0} & \cdots & \mathbf{0} \\
\mathbf{0} & & \mathbf{I} & & & \\
\vdots & & & \ddots & & \vdots \\
\mathbf{0} & \ldots & \ldots & \mathbf{0} & \mathbf{I} & \mathbf{0}
\end{array}\right] .
$$

DEFINITION 1.8. A recurrence (1.20) is (strictly) stable if and only if (1.21) is (strictly) stable.

When $\rho(M)=1$ in (1.21) it is necessary for stability to ensure that the largest eigenvalues of (1.21) are semi-simple. We state the following result without proof.

LEMMA 1.9. Let $\mathbf{M}(\lambda h)$ be given by (1.21); then an eigenvalue $\mu$ is semi-simple if and only if it is a semi-simple zero of the auxiliary polynomial

$$
\operatorname{det}\left\{\sum_{l=0}^{m} \mathbf{X}_{l}(\lambda h) \mu^{m-l}\right\}
$$

in the sense that exactly $\nu$ linearly independent vectors $\zeta$ satisfy $\left\{\Sigma_{l=0}^{m} \mathbf{X}_{l}(\lambda h) \mu^{m-1}\right\} \zeta=0$ where $\nu$ is the multiplicity of $\mu$ as a zero of the auxiliary polynomial. If (1.20) is a scalar recurrence, $\mu$ is semi-simple if and only if it is simple. 
Since stability of recurrence relations is related to the location of zeros of $\operatorname{det}(\mathbf{M}-\mu \mathbf{I})$ or (1.22) we recall the following definitions.

DeFINITION 1.10. A polynomial is said to be a von Neumann polynomial if its zeros lie on the closed unit disk centred on the origin, and its zeros of modulus unity are semi-simple ${ }^{4}$. A polynomial is Schur if it is a von Neumann polynomial with no zeros of modulus unity.

The amplification matrix (1.19) depends upon $\lambda h$, where $\lambda$ is given in (1.15).

DEFINITION 1.11. The region of (strict) stability of a recurrence (1.18) or (1.20) is the set of values $\lambda h \in \mathbb{C}$ for which the recurrence is (strictly) stable. A region of block-stability (respectively strict block-stability) in some norm \|| $\|$ is the set

$$
\{\lambda h \in \mathbb{C} \mid\|\mathbf{M}(\lambda h)\| \leqslant 1\} \quad \text { (respectively }\{\lambda h \in \mathbb{C} \mid\|\mathbf{M}(\lambda h)\|<1\}) .
$$

We hope to model the properties in Definition 1.6 with corresponding properties of (1.18) or (1.20). This leads us to concepts such as $A$-stability, which we define in Section 3. (Strict) stability of (1.18) is analogous to (asymptotic) stability of (1.15).

\section{Quadrature methods revisited}

Our objective is the analysis of Runge-Kutta methods, but to study mixed quadrature-Runge-Kutta methods we require structured rules (1.2). We can then exploit the connection between (1.15) and (1.16).

Suppose that

$$
K(x, y, f(y))=F(y, f(y)), \quad g^{\prime}(x)=0
$$

so that (1.1) reduces to

$$
f^{\prime}(x)=F(x, f(x)), \quad f(0)=g(0) .
$$

A linear multistep method for (2.2) is defined by the parameters $\left\{\alpha_{k}, \beta_{k}\right\}$ in the formula

$$
\sum_{l=0}^{m} \alpha_{l} \tilde{f}((n-l) h)=h \sum_{l=0}^{m} \beta_{l} F((n-l) h, \tilde{f}((n-l) h)),
$$

and by suitable starting values. Associated with (2.3) are (see Lambert [18]) the first and second auxiliary polynomials

$$
\rho^{*}(\mu)=\sum_{l=0}^{m} \alpha_{l} \mu^{m-l}, \quad \sigma^{*}(\mu)=\sum_{l=0}^{m} \beta_{l} \mu^{m-l} .
$$

\footnotetext{
4 If the polynomial is expressed as a determinant (1.22), semi-simplicity will be interpreted in the sense of the preceding lemma.
} 
The application of (2.3) is equivalent to applying the quadrature method to (1.1) with (2.1), when certain conditions are satisfied.

Definition 2.1. The rules (1.2) are called reducible to the linear multistep method $\left\{\alpha_{l}, \beta_{l}\right\}$ or, simply, $\left(\rho^{*}, \sigma^{*}\right)$-reducible, if for some $n_{0} \geqslant 0$,

$$
\begin{gathered}
\sum_{l=0}^{m} \alpha_{l} \omega_{n+1-l . \nu}=0, \quad \nu=0,1, \ldots, n-m ; \\
\sum_{l=0}^{m} \alpha_{l} \omega_{n+1-l, \nu}=\beta_{n+1-\nu}, \quad \nu=n-m+1, n-m+2, \ldots, n+1,
\end{gathered}
$$

when $n \geqslant n_{0}$, and

$$
\sum_{l=0}^{m} \alpha_{l}=0
$$

Suppose (1.2) are $\left(\rho^{*}, \sigma^{*}\right)$-reducible and consider (1.3) with (2.1). Noting that $\omega_{n j}=0$ if $j>n$ we have

$$
\begin{array}{r}
\sum_{l=0}^{m} \alpha_{l} \sum_{j>0} \omega_{n+1-l, j} F((n+1-l) h, \tilde{f}((n+1-l) h)) \\
=\sum_{l=0}^{m} \beta_{l} F((n+1-l) h, \tilde{f}((n+1-l) h))
\end{array}
$$

for $n \geqslant n_{0}$ and we can deduce (2.3) for $n \geqslant n_{0}+1$, given (2.1).

The Gregory rules [4], [7] reduce to Adams-Moulton multistep formulae; other reducible rules can be generated from given $\rho^{*}, \sigma^{*}$.

EXAMPLE 2.1. If $\omega_{i 0}=\omega_{i t}=\frac{1}{2}, \omega_{i j}=1, j=1,2, \ldots, i-1$, then $\alpha_{0}=-\alpha_{1}=1$, $\beta_{0}=\beta_{1}=\frac{1}{2}$.

A more general property of the weights (1.2) can be exploited. First observe that the lower-triangular array of weights $\omega_{n j}$ can quite commonly be partitioned into square submatrices of each of the same order, $q$, namely

$$
\begin{aligned}
& \mathbf{V}_{0,0} \\
& \left(\omega_{n j}\right)=\vdots \quad \cdot \\
& \begin{array}{lllll}
\mathbf{V}_{l, 0} & \mathbf{v}_{l, 1} & \cdots & \mathbf{V}_{l, l} & \\
\mathbf{V}_{l+1,0} & \mathbf{v}_{l+1,1} & \cdots & \mathbf{V}_{l+1, l} & \mathbf{V}_{l+1, l+1}
\end{array}
\end{aligned}
$$

such that for fixed matrices $\left\{\mathbf{A}_{l}, \mathbf{B}_{l}\right\}_{0}^{m}$ we have (defining $\mathbf{V}_{l, j}=\mathbf{0}$ if $j>l$ )

$$
\sum_{l=0}^{m} \mathbf{A}_{l} \mathbf{V}_{n+1-l, v}=\mathbf{B}_{n+1-\nu} \quad\left(n \geqslant n_{0}^{\prime}\right)
$$


for some $n_{0}^{\prime} \geqslant 0$,

$$
\mathbf{A}_{\nu}=\mathbf{B}_{p}=\mathbf{0}, \quad \nu \notin\{0,1,2, \ldots, m\},
$$

and

$$
\sum_{l=0}^{m} \mathbf{A}_{l} \varepsilon=\mathbf{0}
$$

where $\varepsilon=[1,1, \ldots, 1]^{T} \in \mathbf{R}^{q}$. Relations $(2.8 \mathrm{a}, \mathrm{b}, \mathrm{c})$ correspond to generalizations of $(2.5 \mathrm{a}, \mathrm{b}, \mathrm{c})$. We assume, with little loss, that $n_{0}=n_{0}^{\prime}=0$.

Definition 2.2. The rules (1.2) are called reducible to the $m$-step block-method $\left\{\mathbf{A}_{l}, \mathbf{B}_{l}\right\}_{0}^{m}$, or, simply, block-reducible, if $(2.8 \mathrm{a}, \mathrm{b}, \mathrm{c})$ hold.

A subset of the block-reducible rules (1.2) are those for which (2.7) assumes the form

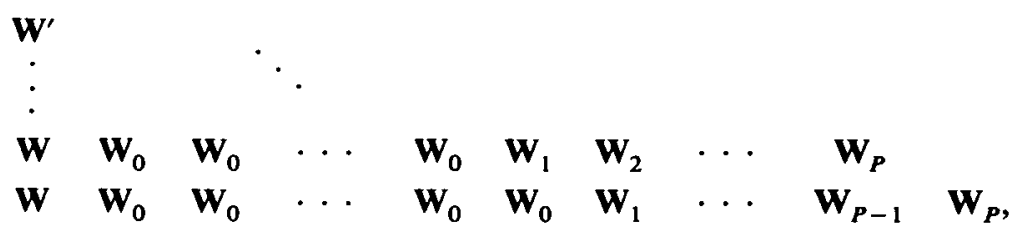

$\begin{array}{llllllllll}\mathbf{W} & \mathbf{W}_{0} & \mathbf{W}_{0} & \cdots & \mathbf{W}_{0} & \mathbf{w}_{0} & \mathbf{W}_{1} & \cdots & \mathbf{W}_{P-1} & \mathbf{W}_{P},\end{array}$

that is, $\mathbf{V}_{l, j}=\mathbf{W}_{P-l+j}$ for $j \geqslant 1$, where $\mathbf{W}_{P+r}=\mathbf{0}, \mathbf{W}_{-r}=\mathbf{W}_{0}$, for $r \geqslant 0$, and $\mathrm{V}_{l, 0}=\mathrm{W}, l \geqslant n_{0}$. All the rules considered by Baker and Keech [7] are of the latter form.

EXAMPLE 2.2. Let the weights (1.2) be defined by equation (1.14) of [7], that is

\begin{tabular}{lccccccc} 
& $j=0$ & 1 & 2 & & $2 s-1$ & $2 s$ & $2 s+1$ \\
\hline$\omega_{2 s, j}=$ & $\frac{1}{3}$ & $\frac{4}{3}$ & $\frac{2}{3}$ & $\ldots$ & $\frac{4}{3}$ & $\frac{1}{3}$ & \\
$\omega_{2 s+1, j}=$ & $\frac{1}{3}$ & $\frac{4}{3}$ & $\frac{2}{3}$ & $\ldots$ & $\frac{4}{3}$ & $\left(\frac{1}{3}+\frac{1}{2}\right)$ & $\frac{1}{2}$.
\end{tabular}

Then $P=1$ and

$$
\mathbf{W}=\left[\begin{array}{cc}
\frac{1}{3} & \frac{4}{3} \\
\frac{1}{3} & \frac{4}{3}
\end{array}\right], \quad \mathbf{w}_{0}=\left[\begin{array}{cc}
\frac{2}{3} & \frac{4}{3} \\
\frac{2}{3} & \frac{4}{3}
\end{array}\right], \quad \mathbf{w}_{1}=\left[\begin{array}{cc}
\frac{1}{3} & 0 \\
\frac{5}{6} & \frac{1}{2}
\end{array}\right] .
$$

The weights $\omega_{i k}$ correspond to the use of the repeated Simpson's rule, with the trapezium rule. 
EXAMPLE 2.3. Let the weights have the structure (2.9) and define $\mathbf{A}_{0}=\mathbf{I}$, $\mathbf{A}_{1}=-\mathbf{I}, \quad \mathbf{A}_{l}=\mathbf{0}, \quad l=2,3, \ldots, P ; \quad m=P$. Then $\mathbf{B}_{0}=\mathbf{W}_{P}, \quad \mathbf{B}_{1}=\mathbf{W}_{P-1}-$ $\mathbf{W}_{P}, \ldots, \mathbf{B}_{P}=\mathbf{W}_{0}-\mathbf{W}_{1}$.

Quadrature rules which reduce to $q$-cyclic linear multistep methods in the sense that

$$
\sum_{l=0}^{m} \alpha_{l}^{(n)} \omega_{n(\mu)-l, \nu}=\hat{\beta}_{n(\mu)-\nu,}^{(\prime)}, \quad \mu \equiv\left\{n(\mu)-n_{0}\right\} \bmod (q)
$$

wherein $\alpha_{l}^{(\mu)}=0$ and $\beta_{l}^{(\mu)}=0$ for $l \notin\{0,1, \ldots, m(\mu)\}, m=\max (m(\mu)$ ), can be identified by the reader (to whom we leave the details) as block-reducible, on writing

$$
\begin{aligned}
& \mathbf{A}_{0}=\left[\begin{array}{llll}
\alpha_{0}^{(0)} & & & \\
\alpha_{1}^{(1)} & \alpha_{0}^{(1)} & & \\
\vdots & \ddots & & \\
\alpha_{q-1}^{(q-1)} & \alpha_{q-2}^{(q-1)} & \cdots & \alpha_{0}^{(q-1)}
\end{array}\right], \\
& \mathbf{A}_{1}=\left[\begin{array}{ccc}
\alpha_{q}^{(0)} & \cdots & \alpha_{1}^{(0)} \\
\alpha_{q+1}^{(1)} & \cdots & \alpha_{2}^{(1)} \\
\vdots & \cdots & \vdots \\
\alpha_{2 q-1}^{(q-1)} & \cdots & \alpha_{q}^{(q-1)}
\end{array}\right]
\end{aligned}
$$

and so on and likewise for $\mathbf{B}_{0}, \mathbf{B}_{1}, \ldots$; see Stetter [22, p. 218].

EXAMPLE 2.4. Consider the weights of Example 2.2. As shown, they can be partitioned as (2.9) and therefore treated as in Example 2.3. Alternatively we may define $\mathbf{A}_{l}, \mathbf{B}_{l}=\mathbf{0}$ if $l>1$ and

$$
\begin{array}{ll}
\mathbf{A}_{0}=\left[\begin{array}{cc}
1 & 0 \\
-1 & 1
\end{array}\right], & \mathbf{A}_{1}=\left[\begin{array}{cc}
0 & -1 \\
0 & 0
\end{array}\right] \\
\mathbf{B}_{0}=\left[\begin{array}{cc}
\frac{1}{3} & 0 \\
\frac{1}{2} & \frac{1}{2}
\end{array}\right], & \mathbf{B}_{1}=\left[\begin{array}{cc}
\frac{1}{6} & \frac{5}{6} \\
0 & 0
\end{array}\right],
\end{array}
$$


or yet again

$$
\begin{array}{ll}
\mathbf{A}_{0}=\left[\begin{array}{cc}
1 & 0 \\
-1 & 1
\end{array}\right], & \mathbf{A}_{1}=\left[\begin{array}{cc}
-1 & 0 \\
0 & 0
\end{array}\right], \\
\mathbf{B}_{0}=\left[\begin{array}{ll}
\frac{1}{3} & 0 \\
\frac{1}{2} & \frac{1}{2}
\end{array}\right], & \mathbf{B}_{1}=\left[\begin{array}{ll}
\frac{1}{3} & \frac{4}{3} \\
0 & 0
\end{array}\right] .
\end{array}
$$

Each formulation corresponds to a recognisable 2-cyclic method.

Since the quadrature methods discussed here reduce to methods for (2.2) when applied with (2.1), the stability of the methods applied to (1.15) can be deduced from results in ordinary differential equations, by reference to $\operatorname{det}\left[\sum_{l=0}^{m}\left\{\mathbf{A}_{l}-\lambda h \mathbf{B}_{l}\right\} \mu^{m-l}\right]$. We refer to Stetter [22].

\section{Stability of Runge-Kutta methods}

The earliest stability studies in the numerical treatment of (1.1) have been concerned with (1.15), namely

$$
f(x)-\lambda \int_{0}^{x} f(y) d y=g(x)
$$

We shall derive criteria for the numerical stability of Runge-Kutta methods for (3.1) and comment on the use of this test equation later. We can distinguish two types of stability.

Definition 3.1. A Runge-Kutta method (1.7a, b) displays full-step stability (when applied to a given test equation) if and only if there exists a stable recurrence between vectors whose components are the values $\tilde{f}(i h) \equiv \tilde{f}_{i(p+1)}$ generated (for $\left.i>n_{0}\right)$. The method likewise displays internal stability if and only if there exists a stable recurrence relation between vectors whose components are successive values $\tilde{f}_{i(p+1)+r+1}, i=n_{0}, n_{0}+1, \ldots \ldots ; r=0,1, \ldots, p$, involved non-trivially.

Strict-stability, and block-stability in a prescribed norm, are similarly defined. Since we seek to model the properties of (3.1) we introduce a concept of $A$-stability (here restricted to full-step values).

Definition 3.2. A Runge-Kutta method $(1.7 \mathrm{a}, \mathrm{b})$ is A-stable if it displays strict full-step stability whenever $\operatorname{Re}(\lambda h)<0$. 
For the methods and test equation considered here, the criteria for full-step and internal stability are broadly the same (though there are distinctions in strict stability); this may not be so when considering other test equations.

We write $\phi_{0}=g(0)[1,1, \ldots, 1]^{T}$ and

$$
\boldsymbol{\phi}_{i+1}=\left[\tilde{f}_{i(p+1)+1}, \tilde{f}_{i(p+1)+2}, \ldots, \tilde{f}_{(i+1)(p+1)}\right]^{T}
$$

for $i=0,1,2, \ldots$, where the values $\left\{\tilde{f}_{j}\right\}$ are defined by a given Runge-Kutta method $(1.7 \mathrm{a}, \mathrm{b})$ applied with $K(x, y, v)=\lambda v$, that is, where

$$
\tilde{f}_{j}-\lambda h \sum_{k>0} \Omega_{j k} \tilde{f}_{k}=g\left(\tau_{j}\right) .
$$

We shall require some additional notation and we write

$$
\mathbf{e}_{0}=[1,0, \ldots, 0]^{T}, \quad \mathbf{e}_{p}=[0,0, \ldots, 0,1]^{T}, \quad \mathbf{e}=[1,1, \ldots, 1]^{T},
$$

and

$$
\mathrm{a}_{p}=\left[A_{p 0}, A_{p 1}, \ldots, A_{p p}\right]^{T}
$$

in terms of the elements of $[\boldsymbol{\theta} \mid \mathbf{A}]$ in (1.10). We also introduce the matrices

$$
\mathbf{A}_{p}=\mathbf{E}_{p} \mathbf{A}, \quad \mathbf{E}_{p}=\mathbf{e e}_{p}^{T} .
$$

Observe that $\mathbf{e}_{p}^{T} \boldsymbol{\phi}_{i+1}=\tilde{f}((i+1) h)$.

\subsection{Extended Runge-Kutta methods}

The extended Runge-Kutta methods are described by Definition 1.4. With the method thus defined in terms of (1.10), the equations (3.3) can be recast using (3.2), and in view of (1.12), as

$$
(\mathrm{I}-\lambda h \mathrm{~A}) \phi_{i+1}-\lambda h \mathrm{~A}_{p} \sum_{k=1}^{l} \phi_{k}=\mathrm{g}_{i+1} \text {. }
$$

Here, $\mathbf{g}_{t+1}=\left[g\left(\tau_{i(p+1)+1}\right), \ldots, g\left(\tau_{(i+1)(p+1)}\right)\right]^{T}$. A recurrence of the form (1.18) is sought: We apply $\mathbf{E}_{p}$ of (3.6) to (3.7) and subtract the result from (3.7), to obtain

$$
(\mathrm{I}-\lambda h \mathrm{~A}) \boldsymbol{\phi}_{i+1}-\mathrm{E}_{p} \boldsymbol{\phi}_{i}=\mathbf{g}_{i+1}-\mathrm{E}_{p} \mathbf{g}_{i}
$$

Excluding the (at most $p+1$ ) exceptional values of $\lambda$ for which $(\mathbf{I}-\lambda h \mathbf{A})$ is singular and for which the method fails, we deduce

$$
\boldsymbol{\phi}_{i+1}=\mathbf{N}(\lambda h) \boldsymbol{\phi}_{i}+\boldsymbol{v}_{i+1},
$$

where $\mathbf{N}(\lambda h)=(\mathbf{I}-\lambda h \mathbf{A})^{-1} \mathbf{E}_{p}$ and $\nu_{i+1}=(I-\lambda h \mathbf{A})^{-1}\left(\mathbf{g}_{i+1}-\mathbf{E}_{p} \mathbf{g}_{i}\right)$. The recurrence (3.9) is of the form (1.18) with amplification matrix

$$
\mathbf{N}(\lambda h)=\mathbf{d}(\lambda h) \mathbf{e}_{p}^{T}
$$


wherein

$$
\mathbf{d}(\lambda h)=(\mathbf{I}-\lambda h \mathbf{A})^{-1} \mathbf{e}
$$

We shall employ $(3.10 a, b)$ later; observe that $N(\lambda h)$ is of rank one, with non-trivial eigenvalue $\hat{\mu}(\lambda h)=\mathbf{e}_{p}^{T} \mathbf{d}(\lambda h)$, that is

$$
\hat{\mu}(\lambda h)=\mathbf{e}_{p}^{T}(\mathbf{I}-\lambda h \mathbf{A})^{-1} \mathbf{e} .
$$

Thus, $\rho(\mathbf{N}(\lambda h))=|\hat{\mu}(\lambda h)|$. Observe also that

$$
\|\mathbf{N}(\lambda h)\|_{\infty}=\|\mathbf{d}(\lambda h)\|_{\infty}, \quad\|\mathbf{N}(\lambda h)\|_{1}=\|\mathbf{d}(\lambda h)\|_{1} .
$$

Conditions for stability and block stability in \|\|$_{\infty}$ and \|\|$_{1}$ for (3.9) now follow.

Analysis of (3.9) is sufficient for internal stability, but applying $\mathrm{e}_{p}^{T}$ to (3.9) yields $\mathbf{e}_{p}^{T} \phi_{i+1}=\mathbf{e}_{p}^{T}(\mathbf{I}-\lambda h \mathbf{A})^{-1} \mathbf{e e}_{p}^{T} \phi_{i}+\mathbf{e}_{p}^{T} \nu_{i+1}$ or, since $\mathbf{e}_{p}^{T} \phi_{i}=\tilde{f}(i h)$,

$$
\tilde{f}((i+1) h)=\hat{\mu}(\lambda h) \tilde{f}(i h)+v_{i+1} \text {, }
$$

where $v_{i+1}=\mathbf{e}_{p}^{T} \nu_{i+1}$. Thus the condition for full-step stability is $|\hat{\mu}(\lambda h)|<1$. The following theorem summarizes the results.

THEOREM 3.3. The extended Runge-Kutta method applied to (3.1) displays internal and full-step stability if and only if $|\hat{\mu}(\lambda h)| \leqslant 1$. For $l=1, \infty$ it displays

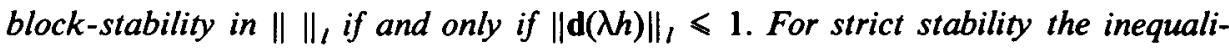
ties must be strict.

In addition we have the following result, using Definition 1.3.

TheOREM 3.4. Let (1.10) be a conventional $R-K$ array. Then the region of stability of (3.9) is the same as the region of stability for the corresponding Runge-Kutta method applied to $y^{\prime}(x)=\lambda y(x), y(0)=y_{0}$.

Proof. See Stetter [22, pp. 131, 174], and observe that $e_{p}^{T} d(\lambda h)$ is the "growth function" of [22] under the given assumption.

COROLlary 3.5. Use of an $A$-stable conventional $R-K$ array yields an extended method which displays $A$-stability.

The following example serves, inter alia, to show that requesting stability (of (3.9)) in a prescribed norm may be too severe.

EXAMPLE 3.1. Consider the conventional $R-K$ tableau

$$
\begin{array}{c|cc}
\frac{1}{2} & \frac{1}{2} & 0 \\
\hline 1 & 1 & 0
\end{array}
$$


We find $\mathbf{d}(\lambda h)=\left[\left(1-\frac{1}{2} \lambda h\right)^{-1},\left(1+\frac{1}{2} \lambda h\right)\left(1-\frac{1}{2} \lambda h\right)^{-1}\right]^{T}$ and $\hat{\mu}(\lambda h)=$ $\left(1+\frac{1}{2} \lambda h\right) /\left(1-\frac{1}{2} \lambda h\right)$ and have $A$-stability. Moreover, (3.9) is strictly block-stable in the uniform norm when $\operatorname{Re}(\lambda h)<0$. In the norm \|\|$_{1}$ this feature is lost, and $\lambda h=0$ is not in the region of block-stability.

3.1.1. We consider the effect of applying the formulae (1.14) rather than (1.12), and write

$$
\mathbf{b}_{p}=\left[B_{p 0}, B_{p 1}, \ldots, B_{p p}\right]^{T}
$$

to find

$$
(\mathbf{I}-\lambda h \mathrm{~A}) \phi_{i+1}-\lambda h \mathbf{e b}_{p}^{T} \sum_{j=1}^{i} \phi_{j}=\mathbf{g}_{i+1}
$$

replacing (3.7). We deduce a relation of the form (3.9) with $\mathbf{N}(\lambda h)$ replaced by

$$
\mathbf{N}_{*}(\lambda h)=\mathbf{N}(\lambda h)+\lambda h \mathbf{d}(\lambda h)\left(\mathbf{b}_{p}^{T}-\mathbf{a}_{p}^{T}\right),
$$

a rank-one matrix whose non-trivial eigenvalue is

$$
\hat{\mu}_{*}(\lambda h)=1+\lambda h \mathbf{b}_{p}^{T}(\mathbf{I}-\lambda h \mathbf{A})^{-1} \mathbf{e} .
$$

For more detail, and an example, refer to [2]. Paul Wolkenfelt observed that a relation similar to (3.13) (with $\hat{\mu}$ replaced by $\hat{\mu}_{*}$ ) can also be found. Thus, the condition for internal and full-step stability is that $\left|\hat{\mu}_{*}(\lambda h)\right|<1$.

\subsection{Mixed quadrature-Runge-Kutta methods with reducible rules}

Amongst the methods of Definition 1.5 are those employing $\left(\rho^{*}, \sigma^{*}\right)$-reducible quadrature rules, satisfying $(2.5 \mathrm{a}, \mathrm{b}, \mathrm{c})$. The scalar equations for the method applied to (3.1) can be re-written in the notation (3.2), (3.6), and in view of (1.13) we obtain

$$
(\mathrm{I}-\lambda h \mathrm{~A}) \phi_{i+1}-\lambda h \sum_{k=0}^{i} \omega_{t k} \mathbf{E}_{p} \phi_{k}=\mathbf{g}_{i+1}, \quad i=0,1,2, \ldots
$$

Thus, when $(\mathbf{I}-\lambda h \mathbf{A})^{-1}$ exists,

$$
\phi_{i+1}-\lambda h \sum_{k=0}^{i} \omega_{i k} \mathbf{N}(\lambda h) \phi_{k}=(\mathbf{I}-\lambda h \mathbf{A})^{-1} \mathbf{g}_{i+1},
$$

employing the matrix (3.10a). We deduce that

$$
\sum_{l=0}^{m} \alpha_{l} \phi_{n+1-l}-\lambda h \sum_{l=0}^{m} \alpha_{l} \sum_{k>0} \omega_{n-l, k} \mathbf{N}(\lambda h) \phi_{k}=\gamma_{n+l},
$$


where $\gamma_{n+1}=\sum_{l=0}^{m} \alpha_{l}(\mathbf{I}-\lambda h \mathbf{A})^{-1} \mathbf{g}_{n+1-l}$, whence, by virtue of $(2.5 \mathrm{a}, \mathrm{b})$,

$$
\sum_{l=0}^{m} \alpha_{l} \phi_{n+1-l}-\lambda h \sum_{l=0}^{m} \beta_{l} \mathrm{~N}(\lambda h) \phi_{n-l}=\gamma_{n+1} \text {. }
$$

The recurrence (3.16) is of the form (1.20) and we deduce the following lemma.

LEMMA 3.6. the mixed quadrature-Runge-Kutta method employing $\left(\rho^{*}, \sigma^{*}\right)$-reducible quadrature displays internal stability if and only if

$$
\operatorname{det}\left[\mu \rho^{*}(\mu) \mathbf{I}-\lambda h \sigma^{*}(\mu) \mathbf{N}(\lambda h)\right]
$$

is a von Neumann polynomial.

For the analysis of full-step stability we apply $\mathbf{e}_{p}^{T}$ to (3.16) to produce, directly on observing that $\mathbf{e}_{p}^{T} \mathbf{N}(\lambda h)=\hat{\mu}(\lambda h) \mathbf{e}_{p}^{T}$,

$$
\sum_{l=0}^{m} \alpha_{l} \tilde{f}((n+1-l) h)-\lambda h \hat{\mu}(\lambda h) \sum_{l=0}^{m} \beta_{l} \tilde{f}((n-l) h)=\mathbf{e}_{p}^{r} \gamma_{n+1} .
$$

Lemma 1.9 yields the following result for full-step stability.

TheOREM 3.7. The mixed quadrature-Runge-Kutta method of Lemma 3.6 exhibits full-step stability when applied to (3.1) if and only if

$$
\mu \rho^{*}(\mu)-\lambda h \hat{\mu}(\lambda h) \sigma^{*}(\mu)
$$

is a von Neumann polynomial. The stability is strict if (3.19) is Schur.

Theorem 3.7 prompts re-examination of (3.17), to relate it to (3.19) under standard assumptions [22, pp. 188, 206].

THEOREM 3.8. Suppose $\rho^{*}(\mu), \sigma^{*}(\mu)$ have no common factors, and $\rho^{*}(\mu)$ is a von Neumann polynomial with $\rho^{*}(1)=0$. The method of Theorem 3.7 applied to (3.1) exhibits internal stability if and only if it exhibits full-step stability.

Remark. We are not in a position to replace "stability" by "strict stability" in the above statement because $\rho^{*}(\mu)$ has roots of modulus unity.

Proof. The value $\mu^{\prime}$ is a zero of (3.17) if and only if, for some $\zeta \neq 0$,

$$
\lambda h \sigma^{*}\left(\mu^{\prime}\right) \mathbf{N}(\lambda h) \zeta=\mu^{\prime} \rho^{*}\left(\mu^{\prime}\right) \zeta .
$$

If $\rho^{*}, \sigma^{*}$ have no common factor, either $\mu^{\prime}=0$ and $\mathbf{N}(\lambda h) \xi=0$ or

$$
\mathbf{N}(\lambda h) \zeta=\mu^{*} \xi
$$

where $\mu^{*}=\mu^{\prime} \rho^{*}\left(\mu^{\prime}\right) /\left\{\lambda h \sigma^{*}\left(\mu^{\prime}\right)\right\}$. For (3.20) to be satisfied, either $\mu^{*}=0$, whence $\rho^{*}\left(\mu^{\prime}\right)=0$, or $\mu^{*}=\hat{\mu}(\lambda h)$ whence $\mu^{\prime}$ must be a zero of (3.19). If $\mu^{\prime}$ is a zero of (3.19) then $\zeta$ is necessarily a multiple of $\mathrm{d}(\lambda h)$ and $\mu^{\prime}$ is semi-simple only 
if it is simple. (If $\mu^{\prime}$ is a zero of $\rho^{*}$ then $\zeta$ is any vector in a $p$-dimensional subspace of vectors annihilated by $\mathrm{N}(\lambda h)$. We expect to find $\rho^{*}\left(\mu^{\prime}\right)=0$ for some $\mu^{\prime}$ with $\left|\mu^{\prime}\right|=1$ and if $\mu^{\prime}$ is a zero of (3.19) then there exist linearly independent vectors $\zeta$ corresponding to $\mu$.)

Example 3.2. Consider the mixed Runge-Kutta method employing the repeated trapezium rule (Example 2.1) and the Runge-Kutta tableau in Example 3.1 , which yields $\hat{\mu}(\lambda h)=\left(1+\frac{1}{2} \lambda h\right) /\left(1-\frac{1}{2} \lambda h\right)$. The polynomials $\rho^{*}(\mu), \sigma^{*}(\mu)$ are determined by the coefficients given in Example 2.1 and the polynomial (3.19) is $\mu(\mu-1)-\frac{1}{2} \lambda h\left(1+\frac{1}{2} \lambda h\right)\left(1-\frac{1}{2} \lambda h\right)^{-1}(\mu+1)$. The zeros of this polynomial are $\mu_{1}=-\frac{1}{2} \lambda h, \mu_{2}=\left(1+\frac{1}{2} h\right) /\left(1-\frac{1}{2} \lambda h\right)$. The method exhibits full-step stability if and only if $\operatorname{Re}(\lambda h)<0$ and $|\lambda h|<2$.

In view of the preceding example we may state the following theorem, which is of some significance.

THEOREM 3.9. A mixed method based on an A-stable reducible quadrature rule and an A-stable Runge-Kutta tableau need not exhibit full-step A-stability.

3.2.1. The previous theorem is disturbing when $A$-stability is a desirable feature, since mixed quadrature-Runge-Kutta methods consume less effort than their extended counterparts. However, the mixed methods can sometimes be modified to obtain $A$-stability. We consider only the economized version of the mixed method, applicable where $\theta_{0}=0$. For this method we replace (3.14) by

$$
(\mathbf{I}-\lambda h \hat{\mathbf{A}}) \phi_{i+1}-\lambda h \sum_{k=0}^{i} \omega_{i k}\left(\mathbf{e}-\mathbf{e}_{0}\right) \mathbf{e}_{p}^{T} \phi_{k}=\mathbf{e}_{0} \mathbf{e}_{p}^{T} \phi_{i}+\left[\mathbf{I}-\mathbf{e}_{0} \mathbf{e}_{0}^{T}\right] \mathbf{g}_{i+1},
$$

where

$$
\hat{\mathbf{A}}=\left[\mathbf{I}-\mathbf{e}_{0} \mathbf{e}_{0}^{T}\right] \mathbf{A}
$$

In consequence

$$
\phi_{i+1}-\lambda h \sum_{k=0}^{i} \omega_{i k}\left[\mathbf{N}_{*}(\lambda h)-\mathbf{N}_{0}(\lambda h)\right] \phi_{k}=\mathbf{N}_{0}(\lambda h) \phi_{i}+\gamma_{i+1}
$$

where $\quad \mathbf{N}_{0}(\lambda h)=[\mathbf{I}-\lambda h \hat{\mathbf{A}}]^{-1} \mathbf{e}_{0} \mathbf{e}_{p}^{T}, \quad \mathbf{N}_{*}(\lambda h)=[\mathbf{I}-\lambda h \hat{\mathbf{A}}]^{-1} \mathbf{e} \mathbf{e}_{\mathrm{p}}^{\mathbf{T}}, \quad$ and $\quad \gamma_{i+1}=$ $[\mathbf{I}-\lambda h \hat{\mathbf{A}}]^{-1}\left[\mathbf{I}-\mathbf{e}_{0} \mathbf{e}_{0}^{T}\right] \mathbf{g}_{i+1}$. Thus,

$$
\begin{aligned}
& \sum_{l=0}^{m} \alpha_{l}\left\{\boldsymbol{\phi}_{n+1-l}-\mathbf{N}_{0}(\lambda h) \phi_{n-l}\right\}-\lambda h \sum_{l=0}^{m} \beta_{l}\left[\mathbf{N}_{*}(\lambda h)-\mathbf{N}_{0}(\lambda h)\right] \phi_{n-l} \\
& =\sum_{l=0}^{m} \alpha_{l} \gamma_{n+l-1} .
\end{aligned}
$$


If we consider full-step stability we take the inner product of $\mathbf{e}_{p}$ and (3.22) to obtain

$$
\begin{aligned}
\sum_{l=0}^{m} \alpha_{l}\left\{\tilde{f}((n+1-l) h)-\mu_{0}(\lambda h) \tilde{f}((n-l) h)\right\} & \\
& -\lambda h \sum_{l=0}^{m} \beta_{l}\left\{\mu_{*}(\lambda h)-\mu_{0}(\lambda h)\right\} \tilde{f}((n-l) h) \\
= & \sum_{l=0}^{m} \alpha_{l} e_{p}^{T} \gamma_{n-l+1},
\end{aligned}
$$

where, in the notation of (3.21) and (3.4)

$$
\mu_{0}(\lambda h)=\mathbf{e}_{p}^{T}[\mathbf{I}-\lambda h \hat{\mathbf{A}}]^{-1} \mathbf{e}_{0}, \quad \mu_{*}(\lambda h)=\mathbf{e}_{p}^{T}[\mathbf{I}-\lambda h \hat{\mathbf{A}}]^{-1} \mathbf{e} .
$$

We deduce the following result.

THEOREM 3.10. Suppose that in the economized mixed quadrature-Runge-Kutta method the rules (1.2) are $\left(\rho^{*}, \sigma^{*}\right)$-reducible. Then the method applied to (3.1) displays full-step stability if and only if

$$
\left\{\mu-\mu_{0}(\lambda h)\right\} \rho^{*}(\mu)-\lambda h\left\{\mu_{*}(\lambda h)-\mu_{0}(\lambda h)\right\} \sigma^{*}(\mu)
$$

is a von Neumann polynomial, and the stability is strict if and only if it is Schur.

EXAMPLE 3.3. Consider the economized version of the method in Example 3.2. The economized method is $A$-stable.

Unfortunately, it is not always true that economized methods have increased regions of stability.

\subsection{Mixed quadrature-Runge-Kutta methods using block-reducible rules}

Consider the methods of Definition 1.5 employing rules (1.2) which satisfy (2.8a, b, c). (For convenience below, $\mathbf{A}_{m+1}=\mathbf{B}_{m+1}=\mathbf{B}_{-1}=\mathbf{0}$ ) We require some additional notation, and we set, for $l=0,1, \ldots, m+1$,

$$
\hat{\mathbf{B}}_{l}=\mathbf{B}_{l-l} \mathbf{J}^{\sharp}+\mathbf{B}, \mathbf{J}
$$

where, in partitioned form,

$$
\begin{aligned}
\mathbf{J} & =\left[\varepsilon_{1}, \varepsilon_{2}, \ldots, \varepsilon_{q-1}, 0\right], \\
\mathbf{J}^{\sharp} & =\left[0,0, \ldots, 0, \varepsilon_{0}\right],
\end{aligned}
$$


and $\varepsilon_{0}, \varepsilon_{1}, \ldots, \varepsilon_{q-1}$ are the successive columns of the identity matrix $\mathbf{I}_{q}$ of order $q$. (We continue to write I for the identity of order $p+1$.) Recall the definition of $\hat{\mu}(\lambda h)$ in (3.11).

The principal result of this section may now be stated.

THEOREM 3.11. The mixed quadrature-Runge-Kutta method employing rules

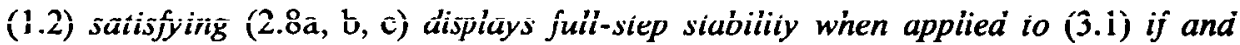
only if

$$
\operatorname{det}\left[\sum_{l=0}^{m+1}\left\{\mathbf{A}_{l}-\lambda h \hat{\mu}(\lambda h) \hat{\mathbf{B}}_{l}\right\} \mu^{m-l+1}\right]
$$

is a von Neumann polynomial; the stability is strict if and only if the polynomial is Schur.

We apply the theorem to an example later but now undertake a proof of the theorem. We define matrices $\hat{\mathbf{V}}_{i j}$ by prescribing the columns $\mathbf{V}_{i j} \boldsymbol{\varepsilon}_{r}=\mathbf{V}_{i j} \boldsymbol{\varepsilon}_{r+1}$ $(r=0,1, \ldots, q-2)$ and $\hat{\mathbf{V}}_{i j} \varepsilon_{q-1}=\mathbf{V}_{i j+1} \varepsilon_{0}$ in terms of (2.7) where $\mathbf{V}_{i j}=\mathbf{0}$ if $j>i$. Thus

$$
\hat{\mathbf{V}}_{i j}=\mathbf{V}_{i j} \mathbf{J}+\mathbf{V}_{i, j+1} \mathbf{J}^{\sharp}
$$

We shall employ the notation for a Kronecker (direct) product between matrices.

Definition 3.12. Let $\mathbf{A}, \mathbf{B}$, be square matrices of order $m, n$ respectively. Then the direct product $\mathbf{A} \otimes \mathbf{B}$ is a matrix of order $m n$ whose $(r, s)$ th submatrix is $A_{r s} \mathbf{B}$, where $A_{r s}$ is the entry in the rth row, sth column, of $\mathbf{A}$.

We set, for $k=0,1,2, \ldots$,

$$
\boldsymbol{\Psi}_{k}=\left[\boldsymbol{\phi}_{k q+1}^{T}, \boldsymbol{\phi}_{k q+2}^{T}, \ldots, \boldsymbol{\phi}_{(k+1) q}^{T}\right]^{T},
$$

where $\phi_{j}$ is defined by (3.2). On reflection (see Example 3.4) we find that the scalar equations defining the method applied to (3.1) can be rewritten as

$$
\left[\mathbf{I}_{q} \otimes(\mathbf{I}-\lambda h \mathbf{A})\right] \boldsymbol{\Psi}_{k}-\lambda h \sum_{j=0}^{k}\left[\hat{\mathbf{V}}_{k j} \otimes \mathbf{E}_{p}\right] \Psi_{j}=\gamma_{k}(\lambda h)
$$

where the components of $\gamma_{k}(\lambda h)$ have the form $g\left(\tau_{\nu}\right)+\lambda h \omega_{\nu, 0} \tilde{f}_{0}$. Since $[\mathbf{A} \otimes \mathbf{B}][\mathbf{C} \otimes \mathbf{D}]=\mathbf{A C} \otimes \mathbf{B D}$, multiplying (3.28) by $\mathbf{I}_{q} \otimes(\mathbf{I}-\lambda h \mathbf{A})^{-1}$ yields 


$$
\boldsymbol{\Psi}_{k}-\lambda h \sum_{j=0}^{k}\left[\hat{\mathbf{V}}_{k j} \otimes \mathbf{N}(\lambda h)\right] \Psi_{j}=\Gamma_{k}(\lambda h)
$$

where $\Gamma_{k}(\lambda h)=\left[\mathbf{I}_{\mathrm{q}} \otimes(I-\lambda h \mathbf{A})^{-1}\right] \gamma_{k}(\lambda h)$.

By virtue of the relations $(2.8 \mathrm{a}, \mathrm{b}, \mathrm{c})$ we find

$$
\sum_{l=0}^{m+1} \mathbf{A}_{l} \hat{\mathbf{V}}_{n+1-l, j}=\hat{\mathbf{B}}_{n+1-j},
$$

where $\hat{\mathbf{B}}_{j}=\mathbf{0}$ if $j \notin\{0,1, \ldots, m+1\}$. It follows that, if we multiply (3.29) by $\mathbf{A}_{l} \otimes \mathbf{I}$ and sum over $l$ we find

$$
\sum_{l=0}^{m+1}\{(\mathbf{A}, \otimes \mathbf{I})-\lambda h \hat{\mathbf{B}}, \otimes \mathbf{N}(\lambda h)\} \Psi_{n+l-l}=\sum_{l=0}^{m+1}\left(\mathbf{A}_{l} \otimes \mathbf{I}\right) \Gamma_{n+1-l}
$$

The auxiliary polynomial for this relation, which allows a study of internal stability, is

$$
\operatorname{det}\left(\sum_{l=0}^{m+1}\left\{\mathbf{A}_{l} \otimes \mathbf{I}-\lambda h \hat{\mathbf{B}}_{l} \otimes \mathbf{N}(\lambda h)\right\} \mu^{m+1-l}\right)
$$

Multiplying (3.31) by $\mathbf{I}_{q} \otimes \mathbf{E}_{p}$ yields, since $\mathbf{E}_{p} \mathbf{N}(\lambda h)=\hat{\mu}(\lambda h) \mathbf{E}_{p}$, the relation

$$
\sum_{l=0}^{m+1}\left\{\left(\mathbf{A}_{l} \otimes \mathbf{E}_{p}\right)-\lambda h \hat{\mu}(\lambda h)\left(\hat{\mathbf{B}}_{l} \otimes \mathbf{E}_{p}\right)\right\} \mathbf{\Psi}_{n+1-l}=\sum_{l=0}^{m+1}\left(\mathbf{A}_{l} \otimes \mathbf{E}_{p}\right) \Gamma_{n+1-l}
$$

Let us now define $\psi_{k}=[\tilde{f}((k q+1) h), \tilde{f}((k q+2) h), \ldots, \tilde{f}((k+1) q h)]^{T}$ where $\tilde{f}((k q+r) h)$ is of course $\mathbf{e}_{p}^{T} \boldsymbol{\phi}_{k q+r}$. Then (3.33) is equivalent to

$$
\sum_{l=0}^{m+1} \mathbf{A}_{l} \psi_{n+1-l}-\lambda h \hat{\mu}(\lambda h) \sum_{l=0}^{m+1} \hat{\mathbf{B}}_{l} \psi_{n+1-l}=\sum_{l=0}^{m+1} \mathbf{A}_{l} \gamma_{n+1-l}
$$

for appropriate vectors $\gamma_{l}$ derived from $\Gamma_{l}$. The stability of (3.34) governs the full-step stability of the method.

EXAMPLE 3.4. Consider the mixed method based on the quadrature weights discussed in Examples 2.2 and 2.4, and the Runge-Kutta tableau discussed in Example 3.1. The tableau of coefficients $\Omega_{j k}$ in (1.7) assumes the form which we partition as shown, the diagonal blocks of order two being the matrix $\mathbf{A}$. The dotted partitioning corresponds to partitioning for the vectors $\phi_{1}, \phi_{2}, \phi_{3}, \ldots$ with the conventions of (3.2) $(f(0)$ being known), whilst the unbroken partitioning corresponds to determination of $\Psi_{1}, \Psi_{2}, \Psi_{3}, \ldots$ defined by (3.27). 


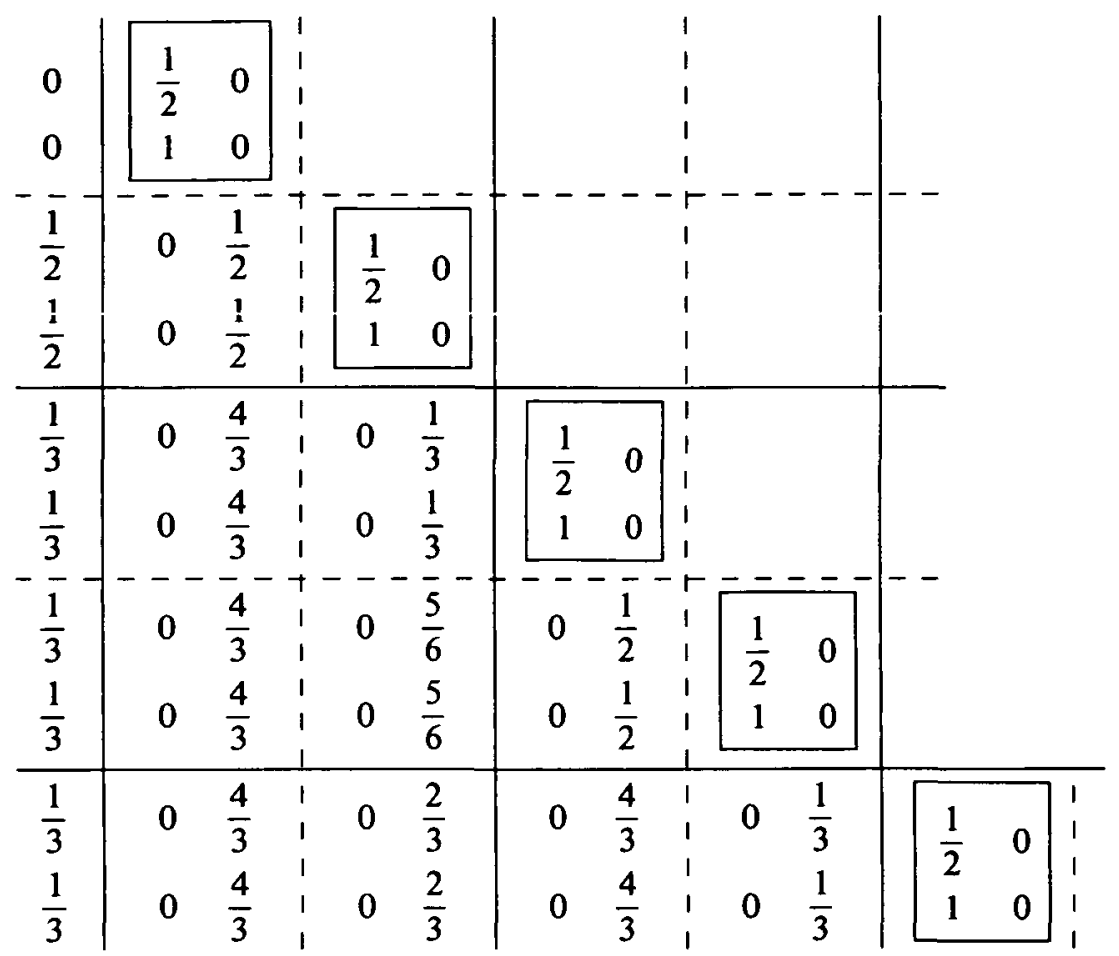

The coefficients of the system of equations (3.29) are obtained as

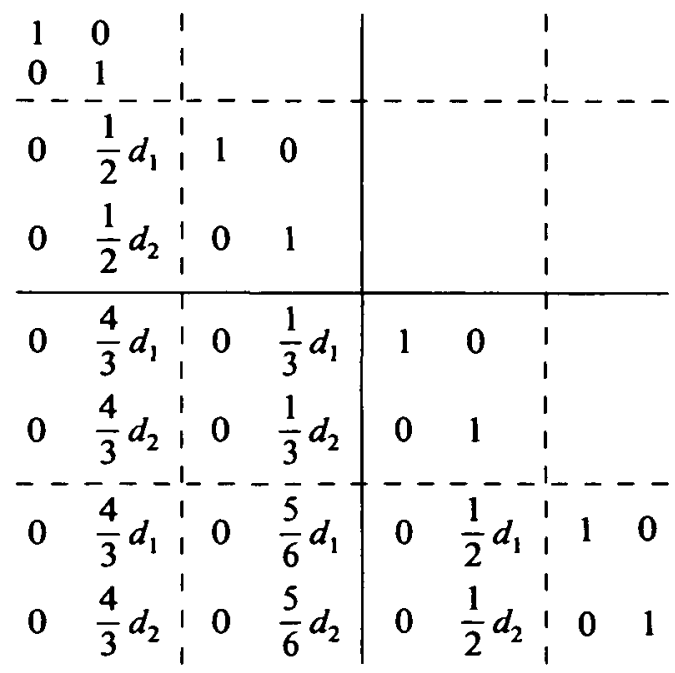

and so on, and a direct approach via differencing allows a stability analysis. For full-step stability the result may be obtained directly by substitution in the determinantal expression given in Theorem 3.11. From Example 2.4 we know 
that we may take $m=1$,

$$
\begin{array}{ll}
A_{1}=\left[\begin{array}{rr}
-1 & 0 \\
0 & 0
\end{array}\right], & A_{0}=\left[\begin{array}{rr}
1 & 0 \\
-1 & 1
\end{array}\right], \\
\mathbf{B}_{1}=\left[\begin{array}{ll}
\frac{1}{3} & \frac{4}{3} \\
0 & 0
\end{array}\right], & \mathbf{B}_{0}=\left[\begin{array}{ll}
\frac{1}{3} & 0 \\
\frac{1}{2} & \frac{1}{2}
\end{array}\right],
\end{array}
$$

which yields

$$
\hat{\mathbf{B}}_{2}=\left[\begin{array}{cc}
0 & \frac{1}{3} \\
0 & 0
\end{array}\right], \quad \hat{\mathbf{B}}_{1}=\left[\begin{array}{cc}
\frac{4}{3} & \frac{1}{3} \\
0 & \frac{1}{2}
\end{array}\right], \quad \hat{\mathbf{B}}_{0}=\left[\begin{array}{cc}
0 & 0 \\
\frac{1}{2} & 0
\end{array}\right] .
$$

From Example 3.1 we know that $\hat{\mu} \equiv \hat{\mu}(\lambda h)=\left(1+\frac{1}{2} h\right) /\left(1-\frac{1}{2} \lambda h\right)$. By Theorem 3.11 we require

$$
\left|\begin{array}{ll}
\mu^{2}-\mu\left(1+\frac{4}{3} \lambda h \hat{\mu}\right) & -\frac{1}{3} \lambda h \hat{\mu} \mu-\frac{1}{3} \lambda h \hat{\mu} \\
-\left(1+\frac{1}{2} \lambda h \hat{\mu}\right) \mu^{2} & \mu^{2}-\frac{1}{2} \lambda h \hat{\mu} \mu
\end{array}\right|
$$

to be a von Neumann polynomial. Denoting its zeros by $\mu_{1}, \mu_{2}, \mu_{3}, \mu_{4}$ we find $\mu_{1}=\mu_{2}=0, \mu_{3} \simeq \frac{1}{6} \lambda h \hat{\mu}, \mu_{4} \simeq 1+2 \lambda h \hat{\mu}$ (and, indeed, we find $\mu_{4}=\exp (2 \lambda h)+$ $O\left(h^{4}\right)$, as might be expected), on substituting the value of $\hat{\mu}$. If $\mu_{3}=\mu_{4}$ we must check for semi-simplicity.

\section{Extensions}

Our analysis, based on the structure of the Runge-Kutta methods, has resulted in recurrence relations and stability polynomials which permit stability results for the "basic" test equation (3.1). We conclude by indicating extensions to the analysis.

Our discussion has been related to the condition $\rho(\mathbf{M})<1$ in recurrences of the form (1.18), resulting in definitions of "absolute" stability. The remarks of Stetter [22, Section 3.5.5] can be paralleled here, and one may seek regions in the $\lambda h$-plane wherein $\rho(\mathbf{M})<\rho^{\prime}, \rho^{\prime}<1$ for $\mathbf{M} \equiv \mathbf{M}(\lambda h)$. Given the nature of the vectors $\Phi_{k}$ in (1.18) and the behaviour of the solution of the test equation it is possible to define relative stability concepts (Baker [4]) which can also be analyzed in terms of the eigenvalues of $\mathbf{M} \equiv \mathbf{M}(\lambda h)$.

The analysis presented here provides a necessary foundation on which to develop a theory for more involved test equations. With regard to the practical 
conclusions which may be drawn from the present work, it is appropriate to issue a caveat. We recall that Baker [4, p. 763] wrote as follows: "It is, of course, the very simplicity of the (test) equation which makes it ideal for mathematical analysis, and a study of this simple case does give some genuine insight .... What we must guard against is an unquestioning acceptance that a method which is suitable for this special equation is suitable for more complicated equations." It now seems to be generally accepted that suitability of a method for the test equation (3.1) is a necessary (but not sufficient) requirement for an all-purpose method.

The structure developed here is of some use in analyzing more general test equations than (3.1) and we note the results in [1], [2] for test equations of the form

$$
f(x)-\int_{0}^{x} \sum_{r=0}^{R} \lambda_{r}(x-y)^{r} f(y) d y=g(x) .
$$

For such equations the concepts of stability and asymptotic stability depend on $R$ and are not covered by Definition 1.6. The appropriate definitions result in generalizing the concept of $A$-stability (applicable to methods for equation (3.1)) to a concept of $(A ; R)$-stability (see [2]), for $R=1,2,3 \ldots A$-stable methods need not be $(A ; R)$-stable, for $R \geqslant 1$.

Other directions in which our analysis can be extended arise when we seek to mimic properties other than those represented by Definition 1.6. Thus, for example, we motivated the definitions of stability and asymptotic stability by classifying the response of $f(x)$ to constant perturbations in $g(x)$. When considering the effect of more general perturbations it is natural to turn explicitly to the properties of, in the linear case, the resolvent kernel, and (for the numerical methods) the inverse of the infinite block-lower-triangular matrix of coefficients in the formulae (1.7) defining the method. The differencing procedure, by which, in the case of (3.1), the latter equations are reduced to the form (1.18), is in effect an eliminaton process permitting such an approach.

Finally let us observe that separable kernels (for example) yield [6] a stability analysis resulting in recurrence relations of the form

$$
\boldsymbol{\Phi}_{k+1}=\mathbf{M}_{k} \boldsymbol{\Phi}_{k}+\gamma_{k}
$$

where strict stability requires $\Pi_{k} \mathbf{M}_{k} \rightarrow \mathbf{0}$. The local stability criterion $\rho\left(\mathbf{M}_{k}\right)<1$, for all $k$, is necessary but not sufficient, whilst the local block-stability criterion $\left\|M_{k}\right\|<1$, for all $k$, is sufficient but not necessary. The analysis for (3.1), wherein $\mathbf{M}_{k} \equiv \mathbf{M}$, provides some insight into which tests are meaningful in the more general case, and the limitations of such tests. We may compare, for example, regions of block-stability in differing norms for the special case, in order to gain insight for the more general case. 


\section{Acknowledgements}

The present paper is based upon the report [2] which contains additional material contributed in part by Siamak Amini. The authors wish to thank Paul Wolkenfelt of Amsterdam, and Siamak Amini and Ian Riddell of Manchester, for their careful reading of the report [2]. One of us (CTHB) wishes also to thank the U. K. Science Research Council for its support.

\section{References}

[1] S. Amini and C. T. H. Baker, "Further stability analysis of numerical methods for Volterra integral equations of the second kind" University of Manchester, Numer. Anal. Tech. Rep. 47 (1980).

[2] S. Amini, C. T. H. Baker, and J. C. Wilkinson, "Basic stability analysis of Runge-Kutta methods for Volterra integral equations of the second kind" University of Manchester, Numer. Anal. Tech. Rep. 46 (1980).

[3] R. Alexander, "Diagonally implicit Runge-Kutta methods for stiff o.d.e.'s" SIAM J. Numerical Analysis 14 (1977), 1006-1021.

[4] C. T. H. Baker, The numerical treatment of integral equations (Clarendon Press, Oxford 1977; second printing 1978).

[5] C. T. H. Baker, Runge-Kutta methods for Volterra integral equations of the second kind (Lecture Notes in Mathematics No. 630 Springer-Verlag, 1978), 1-13.

[6] C. T. H. Baker, "Structure of recurrence relations in the study of stability in the numerical treatment of Volterra integral and integro-differential equations" J. of Integral Equations 2 (1980), 11-39.

[7] C. T. H. Baker and M. S. Keech, "Stability regions in the numerical treatment of Volterra integral equations" SIAM J. Numerical Analysis 15 (1978), 394-417.

[8] C. T. H. Baker, A. Makroglou, and E. Short, "Regions of stability in the numerical treatment of Volterra integro-differential equations" SIAM J. Numerical Analysis 16 (1979), 890-910.

[9] C. T. H. Baker, I. J. Riddell, M. S. Keech, and S. Amini, Runge-Kutta methods with error estimates for Volterra integral equations of the second kind (ISNM Series No. 53 BirkäuserVerlag, Basel, 1980), 24-42.

[10] C. T. H. Baker and J. C. Wilkinson, "Basic stability analysis of Runge-Kutta methods for Volterra integro-differential equations" University of Manchester, Numer. Anal. Tech. Rep. 50 (1980).

[11] B. A. Bel'tyukov, "An analogue of the Runge-Kutta methods for this solution of a nonlinear integral equations of the Volterra type" Differential equations 1 (1965), 417-433 (translation).

[12] J. R. Cash, "Diagonally implicit Runge-Kutta formulae with error estimates" J. Institute of Mathematics and its Applications 24 (1979), 293-301.

[13] J. Donelson III and E. Hansen, "Cyclic composite methods" SIAM J. Numerical Analysis 8 (1971), 137-157.

[14] W. Hahn, Theory and application of Liapunov's direct method (Prentice-Hall, Englewood Cliffs, 1963).

[15] P. J. van der Houwen, Construction of integration formulas for initial value problems (North-Holland, Amsterdam 1977). 
[16] P. J. van der Houwen, "On the numerical solution of Volterra integral equations of the second kind -I Stability; -II Runge-Kutta methods (with J. G. Blom)" Math. Centrum, Amsterdam. Reports NW 42/77 \& $61 / 78$.

[17] P. J. van der Houwen, "Convergence and stability results in Runge-Kutta type methods for Volterra integral equations of the second kind" BIT 20 (1980), 375-377.

[18] J. D. Lambert, Computational methods in ordinary differential equations (Wiley, 1973).

[19] L. Lapidus and J. H. Seinfeld, Numerical solution of ordinary differential equations (Academic, 1971).

[20] K. S. Miller, Linear difference equations (Benjamin, Nẹ York, 1968).

[21] P. Pouzet, "Methode d'integration numerique des equations integrales et integro-differentielles du type de Volterra de seconde espece, Formules de Runge-Kutta" In Sympasium on the numerical treatment of ordinary differential equations, integral equations and integro-differential equations (Birkhäuser, Basel, 1960), 362-368.

[22] H. J. Stetter, Analysis of discretization methods for ordinary differential equations (SpringerVerlag, Berlin, 1973).

[23] Z. B. Tsalyuk, "Volterra integral equations" J. of Soviet Mathematics 12 (1979), 715-758 (in translation).

[24] R. S. Varga, Matrix iterative analysis (Prentice-Hall, 1962).

[25] P. H. M. Wokenfelt, P. J. van der Houwen, and C. T. H. Baker, "Analysis of numerical methods for second kind Volterra equations by embedding techniques" Math. Centrum, Amsterdam, Report NW 71/79 (1979).

\section{Department of Mathematics}

The University

Manchester

England

and

Stanley Park Comprehensive School

Liverpool

England 